\title{
ANALYSIS OF VENTILATION REGIMES OF THE OBLIQUE WEDGE-SHAPED SURFACE PIERCING HYDROFOIL DURING INITIAL WATER ENTRY PROCESS
}

\section{Parviz Ghadimi}

Nasrin Javanmardi

Amirkabir University of Technology, Tehran, Islamic Republic of Iran

\begin{abstract}
The suction side of a surface piercing hydrofoil, as a section of a Surface Piercing Propeller (SPP), is usually exposed to three phases of flow consisting air, water, and vapour. Hence, ventilation and cavitation pattern of such section during the initial phase of water entry plays an essential role for the propeller's operational curves. Accordingly, in the current paper a numerical simulation of a simple surface piercing hydrofoil in the form of an oblique wedge is conducted in three-phase environment by using the coupled URANS and VOF equations. The obtained results are validated against water entry experiments and super-cavitation tunnel test data. The resulting pressure curves and free surface profiles of the wedge water entry are presented for different velocity ratios ranging from 0.12 to 0.64 . Nondimensional forces and efficiency relations are defined in order to present the wedge water entry characteristics. Congruent patterns are observed between the performance curves of the propeller and the wedge in different fully ventilated or partially cavitated operation modes. The transition trend from fully ventilated to partially cavitated operation of the surface piercing section of a SPP is studied and analyzed through wedge's performance during the transitional period.
\end{abstract}

Keywords: emi-submerged propeller; wedge water entry; ventilation pattern; free surface profile.

\section{NOMENCLATURE}

\begin{tabular}{|c|l|}
\hline \multicolumn{2}{|l|}{ Independent Variables: } \\
\hline$D$ & Propeller diameter $(\mathrm{m})$ \\
\hline$\beta$ & Wedge dead-rise angle \\
\hline$n$ & Shaft rotational speed $(1 / \mathrm{s})$ \\
\hline$U$ & Inlet flow velocity $(\mathrm{m} / \mathrm{s})$ \\
\hline$V$ & Wedge vertical velocity $(\mathrm{m} / \mathrm{s})$ \\
\hline$P$ & Pressure $(\mathrm{Pa})$ \\
\hline$P a$ & Ambient pressure $(\mathrm{Pa})$ \\
\hline$P v$ & Saturated vapour pressure $(\mathrm{Pa})$ \\
\hline$\varepsilon$ & Velocity ratio $(-)$ \\
\hline$J$ & Advance coefficient $(-)$ \\
\hline$F r$ & Froude number $(-)$ \\
\hline$R e$ & Reynolds number $(-)$ \\
\hline$\sigma n$ & Cavitation number $(-)$ \\
\hline &
\end{tabular}

\begin{tabular}{|c|l|}
\hline \multicolumn{2}{|l|}{ Dependent Variables: } \\
\hline$T$ & Thrust force $(\mathrm{N})$ \\
\hline$Q$ & Torque $(\mathrm{Nm})$ \\
\hline$K q$ & Torque coefficient $(-)$ \\
\hline$K t$ & Thrust coefficient $(-)$ \\
\hline$K F$ & Force coefficient $(-)$ \\
\hline$\eta p$ & Propeller efficiency $(-)$ \\
\hline$\eta_{\text {wedge }}$ & Wedge efficiency $(-)$ \\
\hline Physical parameters: \\
\hline$\rho s$ & Vapour density $(\mathrm{kg} / \mathrm{m} 3)$ \\
\hline$\rho w$ & Fluid density $(\mathrm{kg} / \mathrm{m} 3)$ \\
\hline$\rho a$ & Air density $(\mathrm{kg} / \mathrm{m} 3)$ \\
\hline$\mu m$ & Mixture viscosity $(\mathrm{kg} / \mathrm{ms})$ \\
\hline$\dot{m}$ & Mass transfer rate $(\mathrm{kg} / \mathrm{s})$ \\
\hline
\end{tabular}




\section{INTRODUCTION}

Nowadays, surface piecing propellers (SPP) are well known for operating in semi-submersible condition at high advance speed (vessel speed greater than 50 knots). The conditions bring about complex ventilation and cavitation patterns to this type of propeller, which have necessitated wide range of research involving experiments and CFD modelling. However, numerical simulation of ventilation and cavitation patterns on this type of propeller still remains a CFD challenge, which can be accomplished through the analysis of a wedge-shape surface-piercing hydrofoil as its blade section.

During the past half-century, considerable number of hydrodynamic researches has been devoted to studying the SPP propeller. Two of the earlier and fundamental researches in this regard were conducted by Hadler (1968) and Hacker (1973). They introduced different partially cavitated and fully ventilated flow patterns for the SPP.

Their experiments indicated that in fully ventilated condition, the ventilated cavity may cover most of the blade's suction side and rotate with the blade. However, by increasing the advance ratio and exceeding a critical value, this regime changes to partially cavitated and as a result the cavity becomes smaller and unstable during blade's rotation. Kruppa (1972) also studied the scaling procedure for the SPP. His experiments revealed that Reynolds and Froude numbers greater than $5 \times 10^{5}$ and 3, respectively, have negligible influence on its scaling. Later, Brandt (1973) developed the primary performance curves of a SPP. Based on his experimental observations, the value of transition advance ratio can be affected by the Cavitation number as well as Froude number. Meanwhile, Olofsson $(1993,1996)$ conducted a wide range of experiments on the surface piercing propeller of 841-B model and recorded transient hydrodynamic forces and torques of a rotating blade. His findings revealed that small Cavitation number is likely to be an effective factor for the transient and average forces. In addition to the above mentioned observations, the value of critical or transition advance ratio of this propeller at different Cavitation numbers was determined.

High speed operation of the SPP requires to use expensive and accurate experimental equipment. To avoid such obstacles, analytical and numerical studies on wedge- shape surface- piercing hydrofoils water entry or in some cases a simplified wedge water entry can be considered suitable alternatives. Yim $(1969,1974)$ is considered a pioneer of the water entry theoretical analysis. He worked out a $2 \mathrm{D}$ potential flow approach for the water entry and exit of a thin infinite symmetric wedge to predict the characteristics of a SPP. His method was later extended by Wang (1977, 1979) to solve finite foil shape problems in fully ventilated condition. By using the conformal mapping method, Khabkkhpasheva (2014) and Ghadimi (2011-2014) studied water entry problems of arbitrary objects. However, this method can be used only for the infinite object water entry and cannot take into consideration the nonlinearity and viscosity of fluid.
Through development and improvement of a numerical method based on lifting-line theory, Furuya (1985) was the first who presented a three-dimensional numerical model for the SPP in fully ventilated operation condition without considering the gravity and blade thickness effects. By using the negative image method, the linearized effect of the free surface was taken into consideration. On the other hand, Wang (1990) conducted a 3D numerical modelling to assess the performance of a fully ventilated SPP by applying an unsteady lifting surface method. Later, Kudo $(1994,1995)$ analyzed the SPP through a 3D lifting surface vortex lattice method (VLM) in submerged condition.

Kinnas and Young $(2002,2003)$ and Young et al. (2011) combined the surface panel method and potential flow based BEM ? to simulate the cavitation sheets on a SPP's blade in submerged and semi-submerged propeller operation. In this approach, cavity borders are assumed to be a boundary of fluid domain and, in order to predict the cavity shape, an iterative procedure is required. Consequently, the blade stable cavitation boundary and mean forces could be obtained from the simulation, but dynamic forces and ventilated free surface profile at entry stage could not be derived precisely. Faltinsen et al. (2008) considered the nonlinearity of the ventilating problem. They solved parametrically the ventilating entry of a semi-infinite flat plate through analytical expressions. Effects of gravity and fluid viscosity were neglected in this investigation. On the other hand, Vinayan (2010) worked out a 2D BEM code to consider the nonlinear nature of water entry process. He obtained the pressure curves and free surface patterns for vertical motion of a symmetric oblique wedge into the calm water and compared his findings with Cox's experiments (1971). Due to the initial usage of calm water condition, the influence of the horizontal water velocity could not be observed. To evaluate the effects of structural deformations on water entry process of a wedge, Maki et al.(2011), Piro and Maki $(2011,2013)$ and Dominic et al. (2013) used coupling elastic beam theory and URANS equations to simulate the flexible wedge water entry. Their studies revealed that for the small structural deformations and moderate dead-rise angle wedge, the effects of structural flexibility is negligible on the water entry process. However in surface piercing sections water entry study by using the coupled URANS and finite element method, Javanmardi and Ghadimi $(2017 \mathrm{a}, 2017 \mathrm{~b})$ showed that the structural deformations of a SPP blade and its hydrofoil sections may have some influence on its water entry process.

Results obtained from the smoothed particle hydrodynamics methods and URANS based on the simulations conducted by Farsi and Ghadimi (2014a, 2014b, 2015), Feizi Chekab et al. (2015), and Shademani et al. $(2016,2017)$, in modelling the arbitrary object's water entry study, showed that considering the effects of fluid viscosity in the numerical simulations improve the accuracy of the flow simulation around the water entering wedge.

The artificial viscosity assumption and ideal fluid consideration in most of the presented numerical simulations have been the cause of some errors in the observed ventilation 
patterns and dynamic forces. Furthermore, most of the numerical schemes have been developed for modelling the fully ventilated operation condition with constant ambient pressure in the ventilated zone. Therefore, the effect of air turbulence on the ventilated cavity has been ignored. In order to obtain more realistic ventilation regimes in different operating conditions such as different advance coefficients, consideration of the effects of some influential physical parameters is imperative. The parameters include fluid viscosity, surface tension, gas dynamics in the ventilated zone as well as growth and collapse of the vapour bubbles. Accordingly, in the current study, unsteady numerical simulations of the ventilating-cavitating turbulent flow around surface piercing hydrofoils in initial water entry phase, are conducted. Flow patterns in different conditions including low, transition, and high advance coefficients, are analyzed. Based on the obtained results, the influence of ventilation and cavitation phenomena on the performance of the SPP are analyzed. To this end, URANS (Unsteady Reynolds Averaged Navier-Stokes) is applied as the governing equations of the turbulent flow. Free surface shape and ventilation patterns are obtained by coupling the URANS and Volume - of- Fluid technique. Since cavitation phenomenon may occur in the initial water entry phase (Brandt (1973)), appropriate cavitation model is employed. In the presence of cavitation, vapour and injected air could coexist in the cavities which represent a multiphase mixture. Therefore, the basic two-phase cavitation model should be replaced by a multiphase model. In the current study, the validated cavitation model developed by Zwart (2004) is employed to capture and assess the cavitation effects on the wedge water entry hydrodynamics.

\section{GOVERNING EQUATIONS}

Since the present study deals with three different phases of water, vapour, and air, the mixture is assumed to be homogeneous. Accordingly, momentum URANS equations are used in terms of mixture's pressure, velocity, and viscosity. In general form, they are:

$$
\begin{aligned}
& \partial\left(\rho_{m} \vec{v}\right) / \partial t+\nabla \cdot\left(\rho_{m} \vec{v} \vec{v}\right)= \\
& \rho_{m} g-\nabla P+\nabla \cdot\left[\mu_{m}\left(\left(\nabla \vec{v}+\nabla \vec{v}^{T}\right)-2 / 3 \nabla \cdot \vec{v} I\right)\right]
\end{aligned}
$$

where $I$ is the unit tensor, $\vec{v}$ and g are velocity and gravity force components, respectively. $P, \mu_{m}$ and $\rho m$ are also the mixture's pressure, viscosity and density, respectively. These parameters can be written in the fractional form of:

$$
\begin{gathered}
\rho_{m}=\sum \alpha_{n} \rho_{n} \\
\mu_{m}=\sum \alpha_{n} \mu_{n}
\end{gathered}
$$

In this equation, $\alpha_{n}$ is the volume fraction of $n$-th fluid in a cell and it is known that:

$$
\sum \alpha_{n}=1
$$

Because of the presence of air in the vapour cavity bubbles, the basic two-phase cavitation model should be replaced by a multi-phase cavitation model. Equations proposed by Singhal (2002), Zwart (2004) and Schnerr (2001) are three available cavitation models which can be used for cavitating turbulence flow simulations. Continuity equation of the mixture can be extended to three modified equations based on the air, water, and vapour fractions. These equations are:

$$
\begin{aligned}
& \partial\left(\alpha_{a} \rho_{a}\right) / \partial t+\nabla \cdot\left(\alpha_{a} \rho_{a} \vec{v}\right)=0 \\
& \partial \alpha_{w} / \partial t+\nabla \cdot\left(\alpha_{w} \vec{v}\right)=-\dot{m} / \rho_{w} \\
& \partial\left(\alpha_{v} \rho_{v}\right) / \partial t+\nabla \cdot\left(\alpha_{v} \rho_{v} \vec{v}\right)=\dot{m}
\end{aligned}
$$

The subscripts $a, v$, and $\mathrm{w}$ refer to the air, vapour, and water components. In these equations, mass transfer occurs only between the water and vapour phase, which is denoted by $\dot{m}$. The value of the parameter depends on the pressure difference between the bubble boundaries and vapour saturation point. Based on this value, two different mass transformation directions, i.e. condensation or evaporation, may occur. Condensation and evaporation mass transfers in Zwart's model is identified by the following equations, respectively:

$$
\dot{m}=\dot{m}_{e}-\dot{m}_{c}
$$

$\dot{m}_{e}=C_{e} \frac{3\left(1-\alpha_{v}\right) \alpha_{n u c} \rho_{v}}{R_{b}} \times \sqrt{2\left(P_{v}-P_{o}\right) / 3 \rho_{w}} \times \operatorname{sign}\left(P_{v}-P_{o}\right)$

$\dot{m}_{c}=C_{C} \frac{3 \alpha_{v} \rho_{v}}{R_{b}} \times \sqrt{2\left(P_{o}-P_{v}\right) / 3 \rho_{w}} \times \operatorname{sign}\left(P_{o}-P_{v}\right)$

According to Zwart's investigation (2004), empirical constants $C_{e}$ and $\mathrm{C}_{c}$ can be set equal to 50 and 0.01 , respectively. Also, the non-condensable vapour fraction, $\alpha_{\text {nuf }}$, and mean radius of bubbles, $R b$, are assumed to be $5 \times 10^{-4}$ and $1 \times 10^{-6}$ $\mathrm{m}$, respectively. The constants are validated and presented by Mejri (2006) and Ji (2011). Moreover, to obtain the free surface pattern, the coupled volume - of - fraction equation is implemented. Since the flow is highly turbulent, the $k-\omega-$ SST turbulent model is applied. 


\section{DEFINITION OF PARAMETERS}

To investigate the hydrodynamic behaviour of the initial water entry process of a SPP, the geometry of the wedgeshaped hydrofoil is taken from a section of 821-b surface piercing propeller. Since the sections with blade radius ranging from 0.5 to 0.7 have the most effective role in the propeller characteristics, the section at $0.55 r$ is considered in this paper, because its shape is also similar to the wedge studied by Cox (1971). The combined principal parameters of the propeller and wedge water entry used in this paper are the advance coefficient $J$, velocity ratio $\varepsilon$, Froude $(F r)$ and Cavitation $\left(\sigma_{n}\right)$ numbers, which are determined by using the following equations, respectively:

$$
\begin{gathered}
J=U / n D \\
V=0.55 \pi n D=0.55 \pi U / J \\
\varepsilon=U / V=J / 0.55 \pi \\
F r=U /(g . D)^{0.5} \\
\sigma_{n}=\left(P_{a t m}-P_{v}\right) / 0.5 \rho_{w} U^{2}
\end{gathered}
$$

In the equations, $D, n, U, V$ and $P_{a t m}$ denote the propeller diameter, shaft speed, water advance speed, foil rotational speed, and ambient pressure, respectively. The geometry of the considered SPP's wedge -shaped hydrofoil is illustrated in Fig. 1.

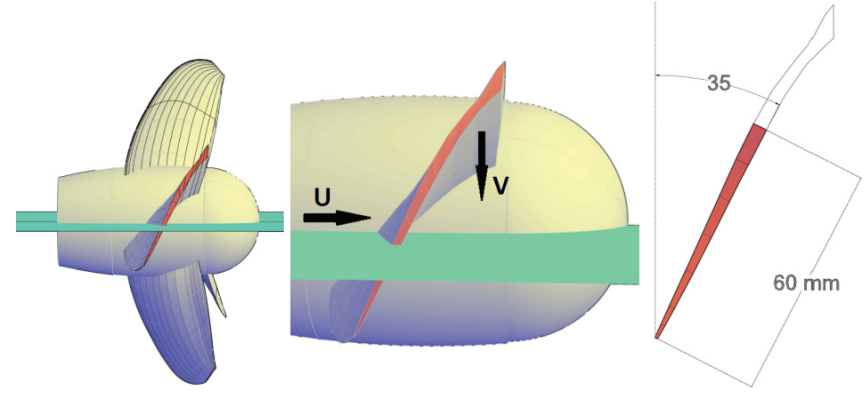

Fig. 1. The hydrofoil section of a SPP @0.55r

In addition to the principal parameters, there are also other dependent parameters which reflect the hydrodynamic performance of the wedge water entry. These parameters include the pressure coefficient, vertical and horizontal forces, and efficiency coefficient. The parameters are determined by using the following equations, respectively:

$$
C_{P}=\left(P-P_{a t m}\right) / 0.5 \rho_{w} V^{2}
$$

$$
K_{F}=F / 0.5 \rho_{w} V^{2} V t
$$

$$
\eta_{\text {wedge }}=F_{x} U / F_{y} V
$$

$$
\eta_{P}=\frac{T U}{2 \pi Q n}
$$

$$
Q=\int F_{y} r d r
$$

In the equations, $K_{F}$ indicates the dimensionless force in $x$ or $y$ direction. $F_{x}$ indicates the wedge thrust force and $F_{y}$ is the force produced by the propeller torque. The wedge efficiency is defined in a similar way as in the propeller efficiency expression $\left(\eta_{p}\right) . T$ and $Q$ denote the propeller thrust force and shaft torque, respectively. The propeller performance is defined on the basis of the thrust and torque coefficients which are given as follows:

$$
\begin{aligned}
& K_{t}=\frac{T}{\rho n^{2} D^{4}} \\
& K_{q}=\frac{Q}{\rho n^{2} D^{5}}
\end{aligned}
$$

The thrust and torque coefficients and efficiency of the above mentioned propeller have been determined in Olofsson's experiments (1993) for Froude number $\mathrm{Fr}=6$ and Cavitation number $\sigma_{n}=2.3$.

\section{VALIDATION OF THE SIMULATED MODEL}

Various investigations have been conducted on the water entry and cavitation problems. Simultaneous presence of the two phenomena in this study requires two types of validations. First validation case is related to the water entry problem. For this case, the results of Cox's experiments (1971), and numerical simulations of Vinayan (2010) of the wedge water entry problem, are used. Second validation case is related to cavitation phenomenon. In this case, results of numerical simulation of a specific super-cavitating foil are compared against its corresponding tunnel test experimental outputs from Dinh's experiments (1968). 


\section{WEDGE WATER ENTRY}

The experiment by Cox (1971) was conducted on the symmetric wedge with dimensions of 0.5 " breadth and 6 " height (c) in initial calm water condition. This wedge profile is the same as the wedge considered in the current paper. The wedge enters the initial calm water with the constant speed $(\mathrm{V})$ of $2.45 \mathrm{~m} / \mathrm{s}$. The free surface profile is investigated for two incident angles of 0 and 10 degrees. The wedge geometry and incident angle definition are illustrated in Fig.2a. The obtained free surface profiles are compared against the results of the experiment by Cox (1971) in Fig. 2 and 3. Also, the pressure distribution for the case $\alpha=10^{\circ}$ is compared with Vinayan's BEM ? results (1971).

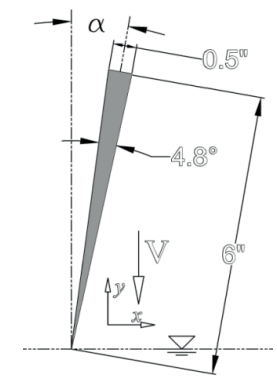

a)

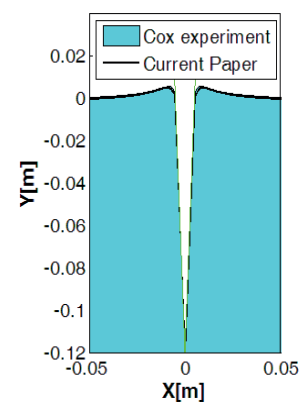

b)
Fig. 2. a) Cox's wedge geometry and b) Comparison of the obtained free surface profile against the result of Cox's experiment (1971) @ $\alpha=0, V=2.45 \mathrm{~m} / \mathrm{s}$.

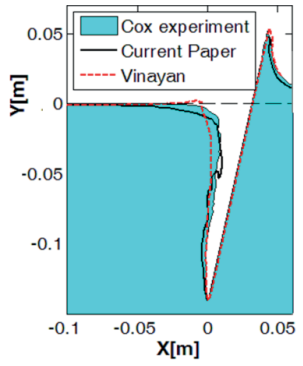

a)

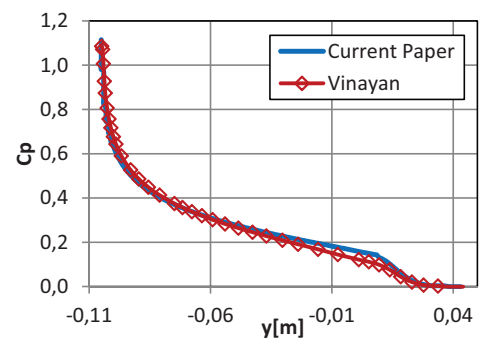

b)
Fig. 3. a) Comparison of the computed free surface profile against the results of Cox's experiment (1971) @ $\alpha=10, V=2.45 \mathrm{~m} / \mathrm{s}, V t / c=0.75$ and $b$ ) Comparison of the computed $C$ against that of BEM model by Vinayan (2010).

As evident in Fig.2b, in the case of symmetric vertical motion the numerical and experimental free surface profiles agree quite well to each other for the incident angle of 0 degree. In the case of 10 degrees incident angle, there is also a favourable agreement between the free surface profiles on the wedge's pressure side, while the profiles agreement on the suction side is relatively good. This is due to the fact that suction side separation profile is very sensitive to: a) edge's apex curvature radius which was not declared in Cox's results (1971) and b) fluid viscosity effects which was ignored in Vinayan's BEM results (2010). Comparison of the computed pressure coefficient $C_{p}$ on the wedge's sides against the results of nonlinear BEM simulation by Vinayan (2010) indicates favourable agreement and it is because of the ignorable effect of viscosity on this side, which has been predicted by Yim (1969).

\section{FLOW AROUND A SUPER-CAVITATING HYDROFOIL}

The super-cavitating flow is numerically simulated around a thin profile through coupled URANS and Zwart (2004) cavitation model equations. 300000 cells are generated in the problem domain. The foil is exposed to the $20 \mathrm{fps}$ fluid flow at the incident angle of $9^{\circ}$ (Fig.4) and Cavitation number of $\boldsymbol{\sigma}=\left(P-P_{v}\right) / 0.5 \rho_{w} U^{2}=0.318$, where $U$ represents the upstream velocity. The foil profile is originally tested by Dinh (1968) in the cavitation test tunnel of Laval University. The geometry of the super-cavitating profile is shown in Fig.4. Comparison of the obtained pressure coefficient $\left(C_{P}=\left(P-P_{o}\right) / 0.5 \rho_{w} U^{2}\right)$ around the foil against Dinh's experimental data (1968) and the results of Wu's analytical model (1956) are presented in Fig.5a. The cavity profile and measured length are also shown in Fig.5b.

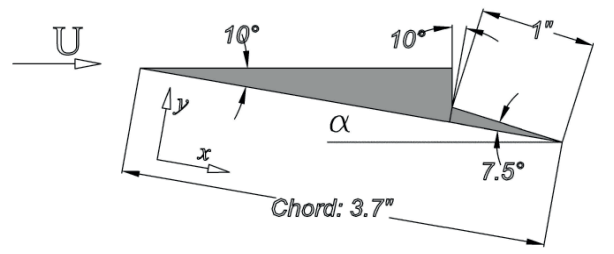

Fig. 4. Geometry of super-cavitating hydrofoil tested by Dinh (1968).

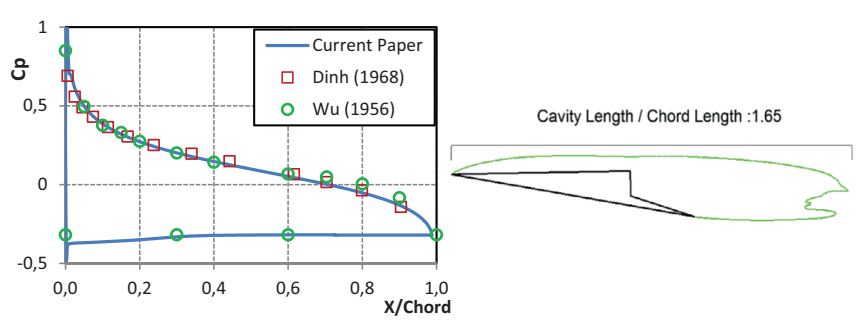

a)

b)

Fig. 5. (a) Comparison of the computed Cp against the results of Dinh's experiment (1968) and Wu's theoretical model (1956) @ $\alpha=9^{\circ}, V=6.15 \mathrm{~m} / \mathrm{s}$ and $\sigma_{n}=0.318$, and (b) Comparison of the obtained vapour cavity profile against Dinh's experimental result (1968).

As evident in Fig. 5, the stagnation point is at the leading edge of the foil and thus the local pressure coefficient at this point is equal to 1 , which is synonymous to the presented numerical result. Furthermore, favourable agreement is observed between the numerically obtained pressure distribution and the results of Dinh's experiment (1968) and Wu's theoretical model (1956). Dinh (1968) recorded the cavity length of $1.8 \times$ chord for the implemented values of input speed, incident angle and ambient pressure. The cavity length obtained in the current study is equal to $1.65 \times$ chord length (as shown in Fig.5b), which indicates $8 \%$ error. It should be noted that radius of hydrofoil's leading edge has also important effects on the generated cavity length. Since this value is unknown it is set to be zero, which leads to some errors. 


\section{SETTING UP THE COMPUTATIONAL PROCEDURE}

In the current study, the geometry of a wedge-shaped surface piercing (SP) hydrofoil with the left dead-rise angle of $120.2^{\circ}$ and right dead-rise angle of $55^{\circ}$ is considered to form a blade section, as shown in Fig.6. Based on the above mentioned dead-rise angles, the pitch angle is found to be $35^{\circ}$. The wedge is situated in a rectangular computational domain containing water and air separated by free surface at a specific height. The computational domain is illustrated in Fig. 6. Initially, the wedge's apex is located at an undisturbed free surface level.
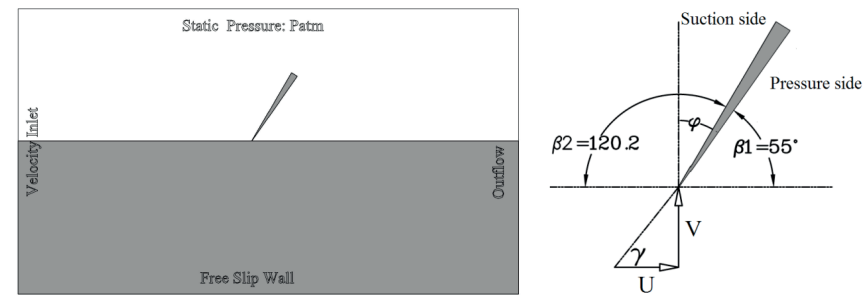

Fig. 6. The considered computational domain and wedge geometry

Similar to the propeller condition, the incoming flow with constant velocity $(U)$ enters from an upstream boundary location. Similar to the Olofsson's experiments (1993), numerical simulation in the current paper is conducted in full-scale condition and the input velocity is set to be $9.39 \mathrm{~m} / \mathrm{s}$. Based on Olofsson's paper (1993), the characteristic curves of 821-b SPP have been presented for the advance coefficients in the range of [0.2 to 1.1]. Therefore, the hydrodynamic characteristics of the wedge water entry is investigated for this range, as well. Accordingly, by using Eq.12, the value of blade velocity $\mathrm{V}$ can be set for each simulation condition. Dimensions of the computational domain are selected based on the work of Shademani and Ghadimi (2016). By considering the gravity effects, distribution of initial hydrostatic pressure based on the water depth is implemented. Downstream location of the computational domain is defined to be an outflow patch, while "no-slip" condition is set for the wedge planes. By adjusting the Courant number below unity, the time step at each blade velocity is prescribed based on the vertical displacement step which is defined by $d y=h / k$. The parameter $h$ is the oblique wedge height and $k$ is the number of divisions assumed in wedge height. The effect of displacement step is assessed by subsequent assuming $k=40,60,120$, and 180. Based on the comparison of the obtained results against Olofsson's experimental data (1993) it is found that decreasing the displacement step slightly affects the computed forces, but strongly influences the free surface smoothness. Therefore, the case of $k=120$ ( $d y \sim 0.5 \mathrm{~mm}$ ) (which shows an error less than $5 \%$ ) is adopted for the targeted analyses in this paper.

A mesh of boundary layer type is used on the wedge sides to improve the accuracy of the predicted wall function, while a structured mesh parallel to the undisturbed free surface level is used for the remaining part of the considered domain. Three zones are formed in the problem domain in dealing with the vertical translation of the wedge : two stationary zones and one moving zone (shown in Fig.7). Dynamic meshing technique is used in the stationary zones because of their moving boundaries (Vinayan, (2009)). The effects of mesh size on the solution are investigated by considering two different meshing options of 0.4 (Vinayan, (2009)) and 0.5 million cells. It is shown that increasing the cell number beyond 0.4 million has less than $5 \%$ effect on the forces. However the mesh option of 0.5 million cells yields a smoother free surface pattern. Accordingly, the mesh option of 0.5 million cells is adopted for the current study. A schematic diagram of the generated mesh is illustrated in Fig.7, where the upper and lower boundaries are kept at constant static pressure with free slip wall conditions. Upstream and downstream boundaries are considered as the inlet and outlet, respectively, as shown in Fig. 6.

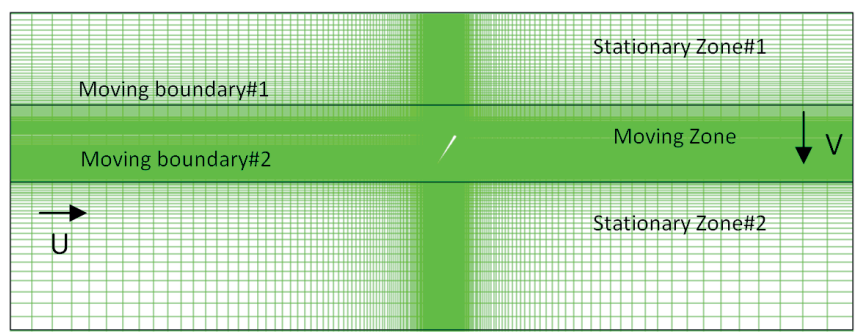

Fig. 7. Illustration of different zones in the domain and the generated mesh

\section{RESULTS AND DISCUSSION}

As previously pointed out in the introduction section, for a constant Froude and Cavitation numbers, the advance coefficient is the only influential parameter for the ventilation pattern. Variation of this parameter leads to the three types of operation conditions. The first subsection (6.1) deals with ventilation pattern and hydrodynamic forces in fullyventilated operation condition, the second subsection (6.2) concentrates on the transition condition, and the third subsection (6.3) focuses on the partial cavitation condition. The fourth subsection (6.4) deals with combining the obtained results in a unified curve. To accomplish this, the required parameters for the targeted analyses are shown in Tab. 1.

Tab. 1. Parameter setting for the performed analyses

\begin{tabular}{|l|c|}
\hline \multicolumn{1}{|c|}{ Parameter } & Value \\
\hline Advance ratio $(J)$ & 0.2 to 1.1 \\
\hline Velocity ratio $(\varepsilon)$ & 0.12 to 0.64 \\
\hline Cavitation number $(\sigma \mathrm{n})$ & 2.3 \\
\hline Froude number $(\mathrm{Fr})$ & 6 \\
\hline
\end{tabular}

\section{FULLY VENTILATED OPERATION CONDITION}

Fully ventilated operation condition deals with a situation in which a single cavity covers the entire wedge suction side (from the leading edge to the trailing edge) during the water entry process. The obtained pressure distributions and free surface patterns for $\varepsilon=0.12-0.29$ are shown in Fig. 8 a and 8 by 
using the dimensionless coordinates $x / V t$ and $y / V t$. Also, the dimensionless exerted forces as well as the defined efficiency of the water entry movement are illustrated in Fig. 8d. The $\mathrm{x}$-component of the cavity wall velocity in the ventilated zone is presented in Fig. 8c.

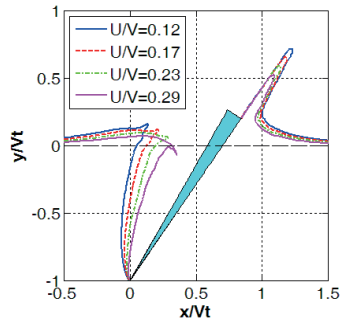

a)

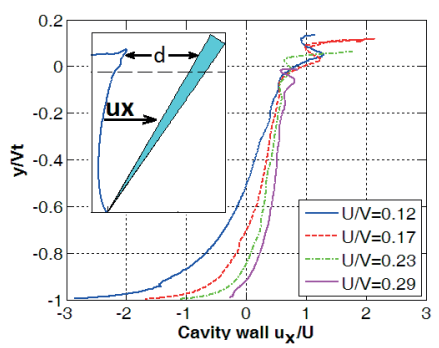

c)

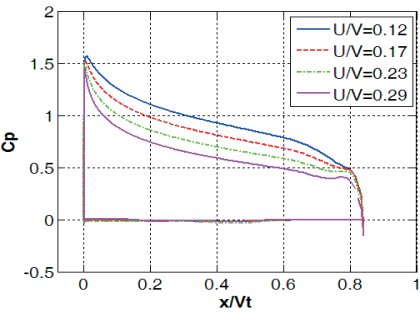

b)

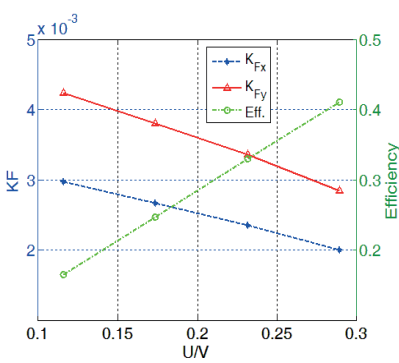

d)
Fig. 8. (a) Free surface profiles, (b) Pressure distributions, (c) Cavity wall velocity $x$-component $u x$, and (d) Dimensionless forces and water entry efficiency@Fr=6, $\sigma_{n}=2.3, \varepsilon=0.12-0.29$.

Based on the numerical results of the free surface profile at the initial stages of water entry (Fig.8a), it can be observed that the pressure drop at the wedge's apex causes an intake of air into the water zone and consequently separation of flow from the wedge apex may occur. The separated flow leads to cavity generation on the wedge's suction side. In this condition, only the pressure side of the wedge interacts with the water, and the resulting force on the wedge is similar to that of the water entry of a flat plate. As evident from the pressure distribution curves of Fig.8b, by increasing the incident angle (decreasing $\varepsilon$ ) at a constant input speed, the mean value of $C_{p}$ increases and thus dimensionless forces increase, as well (Fig.8d). Contrary to this situation, the pressure on the ventilated side does not change significantly from the ambient pressure, and consequently $C_{p}$ tends to zero on this side. Larger vertical blade velocity results in stronger water jet flow on the wedge's pressure side and causes an increase in the lost momentum which reduces the efficiency of water entry process (Fig.8d). Through vertical motion of the wedge, the volume of the ventilated zone gradually increases and, as a result, the atmosphere air is sucked into the cavity from " $d$ " pathway. The width of the pathway depends on the cavity wall velocity in $x$ - direction, $\left(u_{x}\right)$, wedge velocity $V$ and wedge pitch angle. Variation of the magnitude and direction of $u_{x}(\varepsilon, y)$ on the cavity wall for $-1<y / V t<0$ are shown in Fig.8c. It can be seen that as the wedge velocity $V$ reduces the average value of $u_{x}$ increases toward the wedge wall and consequently the volume of the ventilated zone decreases.

\section{TRANSITION CONDITION}

While the propeller rotating speed (wedge vertical speed) declines, the velocity ratio $\varepsilon$ increases and the "full ventilation pattern" is transformed into "transition pattern". This is the unstable and fluctuating state in which its hydrodynamic behaviour highly depends on the wedge geometry, velocity ratio, and ambient pressure. Based on the observations made in the current paper, $\varepsilon$ in the range from 0.36 to 0.42 corresponds to this operational condition. Isolation sequences of the leading edge cavity are shown in Fig. 9a and 9b. Pressure distribution curves are also shown in Fig.10a, while the dimensionless exerted forces and efficiency of the water entry process are illustrated in Fig.10b.

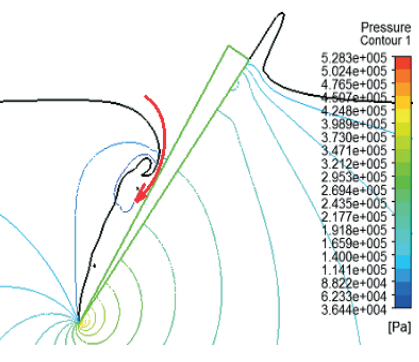

a)

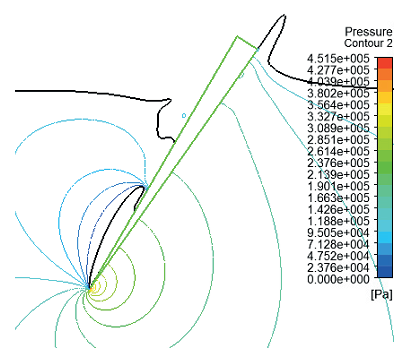

b)
Fig. 9. Ventilated cavity collapse and apex vapour cavity replacement at different velocity ratios: a) $\varepsilon=0.38, b) \varepsilon=0.42$, @ Fr=6 and $\sigma_{n}=2.3$.

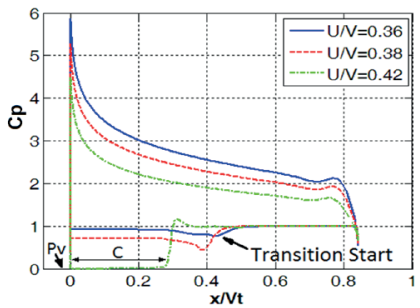

a)

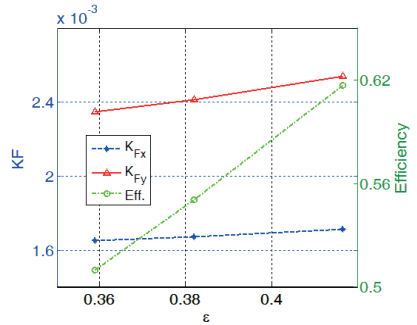

b)
Fig. 10. (a) Pressure distributions, and (b) Dimensionless forces and water entry efficiency@Fr=6, $\sigma_{n}=2.3, \varepsilon=0.36-0.42$.

At a constant water advance velocity, a decrease in the wedge vertical speed would amplify the cavity wall velocity $u_{x}$ toward the wedge ventilated side, which narrows the air pathway " $d$ ", as shown in Fig.8c. The narrowed air pathway causes a pressure drop in the ventilated cavity which can be observed on the pressure distribution curve of $\varepsilon=0.36$ in Fig.10a. The $C_{p}$ variation on the pressure side has the same pattern as in the previous condition. It was pointed out before that the theoretical results can accurately predict the $\mathrm{C}_{\mathrm{p}}$ pattern on this side (Fig.10a). While the vertical speed decreases further, top of the ventilated cavity wall $(y / V t=0)$ collapses through the wedge wall which traps the remaining air near the leading edge. As a result of the vertical displacement of the wedge throughout time and expansion of the cavity wall, the pressure inside the cavity drops. By decreasing the pressure value to that of vapour saturation point in the trapped isolated cavity, water vaporization 
process on the cavity wall begins. The velocity ratio which corresponds to the largest non-ventilated cavity may be called critical velocity ratio. Increasing the forces exerted on the wedge sides in transition condition may be due to the fact that pressure on the wide area of the wedge suction side is less than the ambient pressure and even at critical advance ratio it is equal to vapour pressure (Fig.10b).

\section{PARTIAL CAVITATION CONDITION}

The last type of water entry pattern deals with high velocity ratio condition. The obtained results demonstrate that partially cavitated pattern may happen for the velocity ratio greater than 0.42 . Free surface profile and pressure distribution along the wedge sides for $\varepsilon=0.46 \div 0.64$ are illustrated in Fig.11a, and 11b. Dimensionless exerted forces and water entry efficiency are also shown in Fig.11c.

While the wedge vertical speed (or propeller rotating speed) is very low, the ventilated air cavity collapses in the very initial phase of water entry and only cavitation sheet is observed near the leading edge, whose volume decreases through an increase in $\varepsilon$ value (Fig.11a). Accordingly, the pressure distribution on the wedge pressure side has the same patterns as previously (Figs.11b), while, depending on the cavity length, pressure on the part of the suction side would be equal to the vapour pressure.

Since the dimensionless values of $F x$ and Fy are the function of flow angle of attack ( Fig. 8d and Fig.11c) through an increase of $\varepsilon$, the dimensionless values of $F x$ and $F y$ are reduced in both fully-ventilated and partially-cavitated operation conditions. This is while the efficiency of the blade section increases when $\varepsilon$ rises. This may be attributed to the reduction of the spray height on the pressure side of the wedge and consequently the decrease in the wasted energy as the velocity ratio increases. As a result, the efficiency should increase in both the modes. However, there is an exception in the case of partial cavitation mode, where the impact velocity highly decreases and hence the wetted area increases considerably in such a way that the efficiency drops. Such phenomena can be seen for $\varepsilon=0.64$ (Fig.11c).

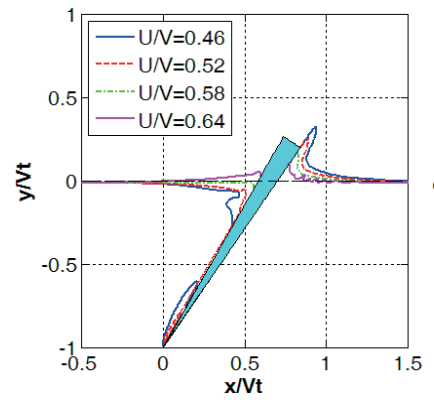

a)

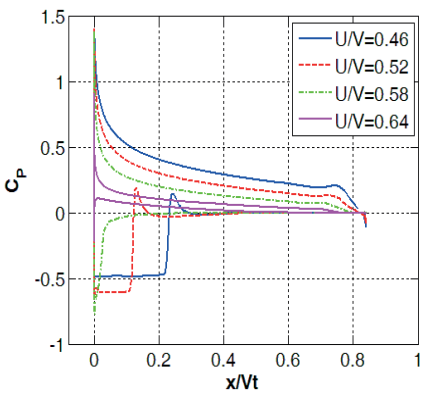

b)

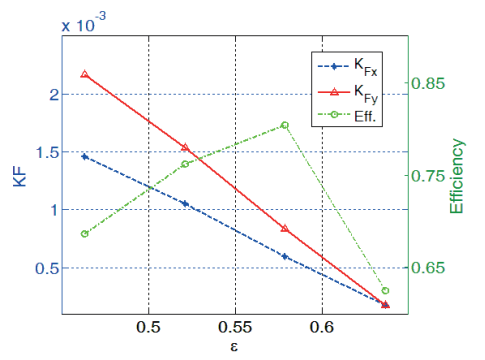

c)

Fig. 11. a) Free surface profiles, b) Pressure distributions, c) Dimensionless forces and water entry efficiency @Fr=6, $\sigma_{n}=2.3, \varepsilon=0.46-0.64$.

\section{INVESTIGATION OF THE PROPELLER AND ITS HYDROFOIL PERFORMANCE CURVES}

The purpose of this subsection is to explore the possible similarity between the performance curve of the studied hydrofoil section and its original SPP. To this end, the results are presented based on the incorporation of wedge water entry and propeller characteristic parameters. For instance, the velocity ratio which is used to evaluate the wedge performance pattern has similar application in the propeller investigation. The experimental curves of 821 -b propeller for $\mathrm{Fr}=6$ have been obtained by Olofsson (1993). Irrespective of the magnitude of $K_{t}$ and $K_{q}$, the overall trends of their corresponding curves are illustrated in Fig.12.

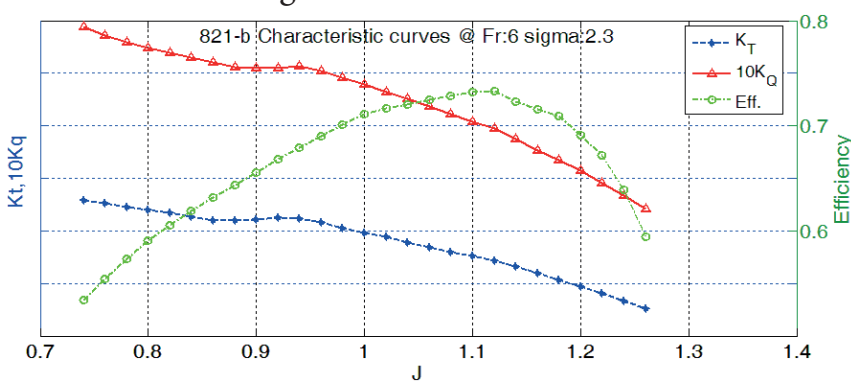

Fig. 12. The characteristic curves of 821-b propeller trend @ Fr=6, from Olofsson's (1993) experiments.

Through combining the results related to forces and efficiency at all three velocity ratios in the unified curves, comprehensive characteristic curves are worked out for the wedge water entry. The resulting curves are illustrated in Fig.13. It should be pointed out that in the curves equivalent value of the advance coefficient is used instead of the velocity ratio on the $x$-axis.

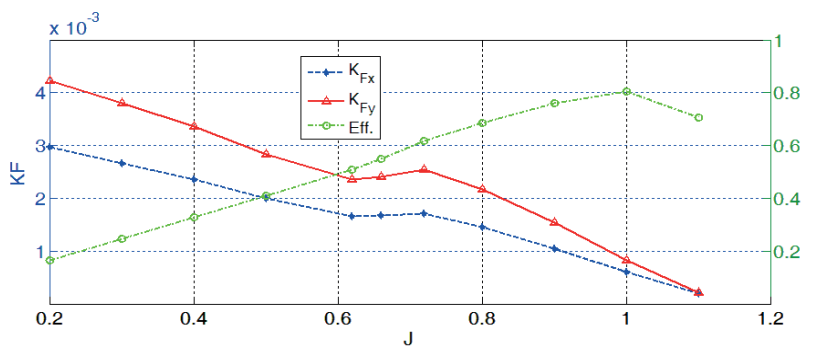

Fig. 13. The obtained characteristic curves for the SP hydrofoil water entry process@Fr=6, $\sigma n=2.3$. 
Comparison of the curves given in Fig.12 and 13 shows that the trends of dimensionless forces and efficiency for both SPP and simplified surface piercing hydrofoil are favourably similar. However, for the studied hydrofoil, the transition pattern occurs at the advance coefficient $(J=0.66)$ lower than that of the SPP 821-b $(J=0.9)$. This discrepancy reveals that transition point for a 3D blade may not be estimated by the simulation of its hydrofoil section. Consequently, estimation of accurate value of transition $J$ for a SPP requires a 3D modelling of the blade water entry. However, this simulation shows that some challenging aspects of SPP's hydrodynamic performance can be recognized through the study of a simple thin wedge water entry.

\section{CONCLUSIONS}

In this paper, hydrodynamic behaviour of a surface piercing hydrofoil is numerically analyzed. The problem is investigated for a multiphase domain containing water, vapour, and air. Coupled URANS equation, VOF scheme, and Zwart's cavitation model (2004) are used to conduct the numerical simulation. The investigated wedge (SP hydrofoil) has the left dead-rise angle of $55^{\circ}$ and right dead-rise angle of $120.2^{\circ}$, which is similar to the Cox's tested wedge (1971). Half a million cells and displacement-step of $0.5 \mathrm{~mm}$ are considered in this simulation. Cox's experimental free surface profiles (1971) are used to validate the water entry simulation part, while Dinh's experimental results (1968) are used for validation of the implemented cavitation model. Favourable accordance is observed in both validation cases between the obtained results and the published experimental data.

Afterwards, water entry process of the wedge is investigated for a particular range of advance coefficients at specific Cavitation and Froude numbers. Three ventilation patterns of fully ventilated, transition, and partially cavitated operation conditions are detected during the period of increasing advance coefficients. Free surface profile and pressure distributions on both sides of the wedge are achieved. The simulation reveals that for specific Froude and Cavitation numbers, the stability and collapse of the ventilated air cavity is highly dependent on the $\mathrm{x}$-component of the cavity wall velocity. It is also found that the magnitude of this velocity is dependent on the wedge velocity ratio.

Similar to the propeller characteristic curves, the wedge's non-dimensional forces and efficiency curves are prepared. Comparison of the characteristics curves illustrates that similar patterns exist between the characteristic curves of the propeller and wedge water entry.

The future research may involve studying the effects of Cavitation number on the transition advance coefficient. This study may become instrumental in better understanding the transition condition regime of the surface piecing propellers.

\section{BIBLIOGRAPHY}

1. 1. Brandt H.: Modellversuche mit Schiffspropellern an der Wasseroberfiache, Schiff und Hafen. 25(5), 1973, pp.415-422.

2. 2. Cox B.D.: Hydrofoil theory for vertical water entry, $\mathrm{PhD}$ thesis, Massachusetts Institute of Technology, Cambridge, MA, USA, 1971.

3. 3. Dinh N.N.: Some Experiments on a Supercavitating Plane Hydrofoil with Jet-Flap, J. Ship Research, SNAME,1968, pp. 207-219.

4. 4. Faltinsen O.M., Semenov Y.A.: Nonlinear problem of flatplate entry into an incompressible liquid. J. Fluid Mech. 611, 2008, pp. 151-173.

5. 5. Farsi M., Ghadimi P.: Finding the best combination of numerical schemes for 2D SPH simulation of wedge water entry for a wide range of dead-rise angles. Int. J Naval Archit. Ocean Eng. 6, 2014, pp. 638-651.

6. 6. Farsi M., Ghadimi P.: Effect of flat deck on catamaran water entry through smoothed particle hydrodynamics. Institution of Mechanical Engineering Part M, J. Engineering for the Maritime Environment, March, 2014, published online.

7. 7. Farsi M., Ghadimi P.: Simulation of $2 D$ symmetry and asymmetry wedge water entry by smoothed particle hydrodynamics method. J. Brazilian Society of Mech. Sci. Eng. 37(3), 2015, pp. 821-835.

8. 8. Feizi Chekab M.A., Ghadimi P., Farsi M.: Investigation of three-dimensionality effects on aspect ratio on water impact of 3 D objects using smoothed particle hydrodynamics method. J. Brazilian Soc. Mech. Sci. Eng, 2015,published online: June 2015. DOI: 10.1007/s40430-015-0367-8.

9. 9. Furuya O.: A performance-prediction theory for partially submerged ventilated propellers. J. Fluid Mechanics, 151, 1985, pp. 311-335.

10. 10. Ghadimi P., Saadatkhah A., Dashtimanesh A.: Analytical solution of wedge water entry by using Schwartz-Christoffel conformal mapping. Int. J. Modeling, Simulation and Scientific Computing. 2(3), 2011, pp. 337-354.

11. 11. Ghadimi P., Dashtimanesh A., Djeddi S.R.: Study of water entry of circular cylinder by using analytical and numerical solutions. J. Brazilian Society of Mech. Sci. Eng. 37(3),,2012, pp. 821-835

12. 12. Ghadimi P., Feizi Chekab MA., Dashtimanesh A.: A numerical investigation of the water impact of an arbitrary bow section. ISH J. Hydraul. Eng. 19(3), 2013, pp. 186-195. 
13. 13. Ghadimi P., Feizi Chekab MA., Dashtimanesh A.: Numerical simulation of water entry of different arbitrary bow sections. J Naval Archit. Marine Eng. 11 (2), 2014, pp. 117-129.

14. 14. Hadler J.B., Hecker R.: Performance of Partially Submerged Propellers, Proc. 7th ONR Symposium on Naval Hydrodynamics, Rome, Italy. 1968.

15. 15. Hecker R.: Experimental Performance of a Partially Submerged Propeller in Inclined Flow, SNAME Spring Meeting, Lake Buena Vista, Florida, USA, 1973.

16. 16. Javanmardi N., Ghadimi P.: Hydroelastic analysis of surface piercing hydrofoil during initial water entry phase, J. Scientia Iranica, accepted to be published, 2017.

17. 17. Javanmardi N., Ghadimi P.: Hydroelastic analysis of a semi submerged propeller using simultaneous solution of Reynolds averaged Navier-Stokes equations and linear elasticity equations, Journal of Engineering for Maritime Environment,? 2017.

18. 18. Ji B., Luo X.W., Wang X., Peng X.X., Wu Y.L., Xu H.Y.: Unsteady numerical simulation of cavitating turbulent flow around a highly skewed model marine propeller. J. Fluids Eng.-Trans, ASME, 133 (1), 011102, 2011.

19. 19. Khabkkhpasheva TI, Kim Y, Korobkin AA.: Generalized Wagner Model of Water Impact by Numerical Conformal Mapping. App. Ocean Res. 44, 2014, pp. 29-38.

20. 20. Kruppa CFL.: Testing of Partially Submerged Propellers, Proc. 13th ITTC Report on Cavitation. Berlin \& Hamburg, Germany, 1972.

21. 21. Kudo T., Ukon Y.: Calculation of super cavitating propeller performance using a vortex-lattice method, in: Second International Symposium on Cavitation, Tokyo, Japan, 1994.

22. 22. Kudo T., Kinnas S.: Application of vortex/source lattice method on supercavitating propellers, in 24th American Towing Tank Conference, College Station, TX, USA, 1995.

23. 23. Maki K.J., Lee D., Troesch A.W., Vlahopoulos N.: Hydroelastic impact of a wedge-shaped body. Ocean Engineering 38, 2011, pp. 621-629.

24. 24. Mejri I., Bakir F., Rey R., Belamri T.: Comparison of computational results obtained from a homogeneous cavitation model with experimental investigations of three inducers, J. Fluids Eng.-Trans. ASME, 128, 2006, pp.1308-1323

25. 25. Olofsson N.: A Contribution on the Performance of Partially Submerged Propellers, Proc. FAST '93, 2nd Intl.
Conf. on Fast Sea Transportation. Yokohama, Japan, 1, 1993, pp. 765-776.

26. 26. Olofsson N.: Force and Flow Characteristics of a Partially Submerged Propeller, PhD Thesis, Department of Naval Architecture and Ocean Engineering, Chalmers Univ., Gotenborg, Sweden,1996.

27. 27. Piro D.J., Maki K.J.: Hydroelastic Wedge Entry and Exit .11th International Conference on Fast Sea Transportation FAST 2011, Honolulu, Hawaii, USA, September 2011.

28. 28. Piro D.J., Maki K.J.: Hydroelastic analysis of bodies that enter and exit water. Journal of Fluids and Structures 37, 2013, pp. 134-150.

29. 29. Panciroli, R.: Water entry offlexible wedges: Some issues on the FSI phenomena. App. Ocean Res. 39, 2013, pp.72-74.

30.30. Schnerr G.H., Sauer J.: Physical and numerical modeling of unsteady cavitation dynamics, in: Proceeding of 4th International Conference on Multiphase Flow, New Orleans, USA, 2001.

31. 31. Shademani, R., Ghadimi P.: Estimation of water entry forces, spray parameters and secondary impact of fixed width wedges at extreme angles using finite element based finite volume and volume of fluid methods. J. Brodogradnja 67(2), 2016, pp. 101-124.

32. 32. Shademani, R., P. Ghadimi.: Asymmetric Water Entry of Twin Wedges with Different Deadrises, Heel Angles, and Wedge Separations using Finite Element Based Finite Volume Method and VOF. Journal of Applied Fluid Mechanics, 10(1), 2017, pp. 353-368.

33. 33. Shademani R., Ghadimi P.: Numerical assessment of turbulence effect on forces, spray parameters, and secondary impact in wedge water entry problem using $k-\varepsilon$ method, Scientia Iranica- B, 24(1), 2017, pp. 223-236.

34. 34. Singhal A.K., Athavale M.M., Li H., Jiang Y.: Mathematical basis and validation of the full cavitation model, J. Fluid Engineering, 124, 2002, pp. 617-624.

35. 35. Vinayan V., Kinnas S.A.: A numerical nonlinear analysis of two-dimensional ventilating entry of surface-piercing hydrofoils with effects of gravity, J. Fluid Mech. 658,2010, pp. 383-408 .

36. 36. Vinayan V. A Boundary Element Method for the Strongly Nonlinear Analysis of Ventilating Water-entry and Wavebody Interaction Problems. PhD thesis, Ocean Engineering Group, Architectural and Environmental Engineering, University of Texas at Austin, Austin, TX, USA, 2009. 
37. 37. Wang D.P.: Water entry and exit of a fully ventilated foil, J. Ship Res. 21 (1), 1977, pp. 44-68.

38. 38. Wang D.P.: Oblique water entry and exit of a fully ventilated foil, J. Ship Res, 23, 1979, pp. 43-54.

39. 39. Wang G., Zhu X., Sheng Z.: Hydrodynamic forces of a three-dimensional fully ventilated foil entering water. J. Hydrodynamics, 5(2), 1990.

40. 40. Wu T.Y.: A Free-Streamline Theory for Two-Dimensional Fully Cavitated Hydrofoils, Mathematical Physics, 35, 1956, pp. 236-265.

41. 41. Yim B.: An application of linearized theory to water entry and water exit problems. Part 2 with ventilation, Rep. 3171. NSRDC, Washington, DC, USA. 1969.

42. 42. Yim B.: Linear theory on water entry and exit problems of a ventilating thin wedge, J. Ship Res. 18 (1), 1974, pp. 1-11.

43. 43. Young Y.L.: Numerical modeling of supercavitating and surface-piercing propellers, $\mathrm{PhD}$ thesis, Ocean Engineering Group, Department of Civil ?, University of Texas at Austin, Austin, TX,USA, 2002.

44. 44. Young Y.L., Kinnas S.A.: Analysis of supercavitating and surface-piercing propeller flows via BEM, J. Computational Mechanics, 32, 2003, pp. 269-280.

45. 45. Young Y.L., Savander B.R.: Numerical analysis of largescale surface-piercing propellers. J. Ocean Engineering, 38, 2011, pp. 1368-1381.

46. 45. Zwart P., Gerber A.G., Belamri T.: A Two-Phase Model for Predicting Cavitation Dynamics, ICMF International Conference on Multiphase Flow, Yokohama, Japan, 2004.

47. Plik : PMR-2016-00084 : 43230 zn. norm. [24 str], stan 2018-01-16, kor. ang. epw

\section{CONTACT WITH THE AUTHORS}

Parviz Ghadimi

e-mail:pghadimi@aut.ac.ir

Amirkabir University of Technology

424 Hafez Ave., 3314 Tehran

ISLAMIC REPUBLIC OF IRAN

Nasrin Javanmardi e-mail:n.javan@aut.ac.ir Amirkabir University of Technology 424 Hafez Ave., 3314 Tehran ISLAMIC REPUBLIC OF IRAN 